\title{
Impact of a Nurse Manager Peer Mentorship Program on Job Satisfaction and Intent to Stay
}

\author{
Tonya Roth, DNP, RN NE-BC \\ Kaiser Permanente, Oregon, United States
}

Diane Whitehead, EdD, DNP, RN, ANEF

Walden University, Minnesota, United States

Contact: troth0907@gmail.com

\begin{abstract}
Nurse managers (NMs) play a vital role in patient outcomes by providing a stable work environment for teams. Numerous factors influence a NM's job satisfaction and intent to remain in a job. The purpose of this project was to develop an evidence-based formal mentorship program for NMs in an effort to impact retention rates. A secondary purpose was to evaluate the impact that a formal mentorship program has on NMs' job satisfaction and intent to stay. Across two hospitals in the Pacific Northwest, 15 NMs participated in a 6month mentorship program. The program was guided by both the mentorship enactment theory and Kouzes and Posner's exemplary leadership framework. Using the Leadership Practices Inventory and the Nurse Manager Practice Environment scale, job satisfaction, intent to stay in a job, and transformational leadership behaviors were measured before and after the program, Results were analyzed using a paired-samples $t$ test. There were statistically significant differences between the preprogram Leadership Practices Inventory scores $(M=212.27, S D=37.8)$ and postprogram scores $(M=232.47, S D=25.28) ; t(14)=-2.83, p=.013$. There were also statistically significant differences between the preprogram Nurse Manager Practice Environment Scale-Culture of Generativity subscale $(M=23.20, S D=4.65)$ and post-program scores $(M=26.20, S D=$ $4.74) ; t(14)=-2.40, p=.032$. The results demonstrated a significant increase in job satisfaction, intent to stay, and transformational leadership behaviors. Implementation of this pilot program supported positive social change through reduced NM turnover, resulting in a reduction of healthcare spending on replacement costs in addition to improved patient outcomes.
\end{abstract}

Keywords: mentorship; mentoring; job satisfaction; retention; nurse manager

Date Submitted: May 31, 2019 | Date Published: October 2, 2019

\section{Recommended Citation}

Roth, T., \& Whitehead, D. (2019). Impact of a nurse manager peer mentorship program on job satisfaction and intent to stay. Journal of Excellence in Nursing and Healthcare Practice, 1, 4-14. https://doi.org/10.5590/JENHP.2019.1.1.02 


\section{Introduction}

Nurse managers (NMs) play a pivotal role within a hospital. Studies have shown a positive correlation between transformational leaders and a variety of patient outcomes (Wong, 2015), and turnover in this role has been shown to have an impact on nurse-sensitive indicators such as falls and pressure ulcers (Warshawsky, Rayens, Stefaniak , \& Rahman, 2013). NMs are responsible for numerous direct reports, larger budgets, clinical outcomes, and 24-hr operations. These tall demands can lead to higher burnout levels and turnover within the NM role (Hewko, Brown, Fraser, Wong, \& Cummings, 2015). Within the state of Oregon, the NM role in a hospital was cited to be the most challenging role to fill when vacant: $61.9 \%$ of employers ranked it very difficult to fill in 2016 (Oregon Center for Nursing, 2016). Furthermore the cost to replace a NM was estimated to be anywhere between $\$ 132,000$ and $\$ 228,000$ (Sherman, Patterson, Avitable, \& Dahle, 2014). NM retention and job satisfaction are complex phenomena to understand. Throughout the literature, there have been suggestions to address span of control, leadership competencies, work-life balance, and mentorship in accentuating the vital role of the NM. To date, there has been no set of best practices identified to scientifically approach this practice problem; however, there does seem to be a common suggestion of mentorship as a suggested intervention (DeCampli \& Nash, 2015; Rich et al., 2015; Westcott, 2016). The purpose of this project was to develop an evidence-based formal mentorship program for NMs in an effort to impact retention rate at two hospitals in the Pacific Northwest. A secondary purpose was to evaluate the impact that a formal mentorship program has on NM job satisfaction and intent to stay.

\section{Background}

To understand retention, one needs to first understand what influences a NM's job satisfaction. Factors that support job satisfaction within the role of NM may differ from staff nurses' role (Djukic, Jun, Kovner, Brewer, \& Fletcher, 2017), and often, the complexity of the NM work environment plays a significant part in a NM's intent to leave (Warshawsky, Wiggins, \& Rayens, 2016) . To navigate the complex work environment, Cziraki, McKey, Peachey, Baxter, and Flaherty (2014) strongly recommended that all new NMs are assigned a mentor. Mentorship was often cited as a recommendation to support NMs, and successful programs such as the Organization of Nurse Executives of New Jersey statewide mentorship program (Rich et al., 2015) demonstrate through attendance the hunger that NMs have for formal programs such as this.

Miltner, Jukkala, Dawson, and Patrician (2015) further uncovered the professional development needs of NMs and found that managers could benefit from additional higher level leadership competencies and formalized mentorship or coaching. Interestingly, Cadmus and Johansen (2012) proposed developing a NM residency program similar to the evidence-based approach for new graduate nurses in an effort to successfully transition nurses into the manager role. However, as more attention is focused on the role of NMs, evidence has shown that NMs still often lack formal role orientation and mentorship (Keys, 2014; Weaver-Moore, Sublett, \& Leahy, 2016). Furthermore, there appeared to be a gap in the literature demonstrating whether formal mentorship has an impact on NM job satisfaction and intent to stay despite it often being recommended.

\section{Theoretical Framework}

Both mentoring enactment theory (MET) and Kouzes and Posner's (2017) framework titled "The Five Practices of Exemplary Leaders" were used in developing the NM mentorship program. MET is an established theoretical framework that describes communication strategies used in successful mentorship relationships. Kalbfleisch (2002) believed the mentoring relationship was a personal relationship between a more seasoned individual and a less seasoned protégé and together, they formed a relationship that included caring and assistance (Kalbfleisch, 2002). The first five propositions of MET are focused on the initiation of the 
relationship, which was used throughout the program to guide relationship building between the mentor and mentee (Kalbfleisch, 2008). Kalbfleisch (2002) postulated that a mentor is more likely to become involved in a mentoring relationship if he or she is approached ahead of time by a third party as opposed to being sought out initially by a protégé. The MET focuses on strategies for successful initiation of the relationship and strategies for maintaining it. Both of these strategies are important to understanding the nature of a healthy mentorship relationship and have been used to evaluate relationships such as that between the doctoral student and advisor (Mansson \& Myers, 2012). Frontline NMs need a unique mentorship relationship to support and guide them in their nurse leadership practice and skills development. This relationship requires that the mentor had a previous successful NM experience and was willing to serve as a mentor. For both the mentor and mentee, trust is critical, as well as the understanding that the relationship may evolve or resolve depending on the needs of the NM. These MET concepts were used to develop the NM mentorship relationship for the program.

Transformational leadership is often described as the golden standard for leadership behaviors, and the theory has been used in a multitude of organizational settings including healthcare (Fischer, 2016). In nursing, transformational leadership has been associated with increased nurse satisfaction and thus staffing retention (Kelly, Wicker, \& Gerkin, 2014) as well as the potential positive impact on patient outcomes. Kouzes and Posner (2017) defined five best practices of transformational leadership: (a) model the way, (b) inspire a shared vision, (c) challenge the process, (d) enable others to act, and (e) encourage the heart. The Formal Nurse Manager Mentorship Program (FNMMP) used the most current edition of Kouzes and Posner's exemplary leadership framework to guide the development of the mentorship program itself to focus the relationship on transformational leadership behaviors and support successful matching of mentorship dyads.

\section{Method}

\section{Purpose}

The main purpose of this project was to develop an evidence-based formal mentorship program for NM's in an effort to increase retention rates at two hospitals in the Pacific Northwest. A secondary purpose was to evaluate the impact that a formal mentorship program has on NM job satisfaction and intent to stay.

\section{Setting}

The FNMMP took place at two hospitals within the same health system in the Pacific Northwest.

\section{Participants}

NMs were recruited on a voluntary basis from two hospitals. There was no minimum number of participants identified for the project. Participants were required to have at least 9 months of experience in their role as a $\mathrm{NM}$ and not be enrolled in the organization's new manager academy. Recruitment involved an emailed invitation to the NMs at both hospitals to participate in a 6-month-long program focused on formal mentorship of transformational leadership behaviors. Initially, $17 \mathrm{NMs}$ out of 43 volunteered to participate in the FNMMP and 15 NMs completed the program. Two NMs withdrew after the first session for personal reasons. Table 1 describes the nursing and management experience, highest nursing degree experience, and current years in the NM position of the participating NMs. 
Table 1. Descriptive Summary of the Participating Nurse Managers ( $\mathrm{N}=15)$

\begin{tabular}{lcc}
\hline Statistic & \% & $\boldsymbol{n}$ \\
\hline Nursing experience, years & & \\
$6-11$ & 13.3 & 2 \\
$12-16$ & 46.7 & 7 \\
$17-22$ & 13.3 & 2 \\
$23-28$ & 20.0 & 3 \\
$\geq 29$ & 6.7 & 1 \\
Management experience, years & & \\
$\quad<2$ & 53.3 & 8 \\
$2-7$ & 40 & 6 \\
$\geq 18$ & 6.7 & 1 \\
Highest nursing degree & & \\
Associate & 13.3 & 2 \\
Bachelor & 60.0 & 9 \\
$\quad$ Master & 26.7 & 4 \\
Outside management experience & & \\
$\quad$ Yes & 13.3 & 2 \\
No & 86.7 & 13 \\
Years in current position & & \\
$\quad<2$ & 66.6 & 10 \\
$2-7$ & 26.7 & 4 \\
$8-12$ & 6.7 & 1 \\
\hline
\end{tabular}

\section{Ethical Considerations}

Participation in the mentorship program was voluntary. Interested participants received details regarding program length, participant expectations, and time required for formal mentorship activities. Participants were allowed to withdraw from the program at any time. To maintain privacy during data collection, each participant used an assigned code number when completing the Nurse Manager Practice Environment (NMPE) scale and the Leadership Practices Inventory (LPI). Walden University Institutional Review Board approval (Number 02-28-18-0731509) was obtained prior to implementing the mentorship program. In addition, a letter of cooperation for the organization was also obtained to include both hospitals. Participants signed a consent form to participate in anonymous questionnaires.

\section{Program Design}

The project had a quasi-experimental, one-group pretest/posttest design. The FNMMP took place over the course of 6 months, with the NMs meeting as a cohort for $2 \mathrm{hr}$ each month. Participants were given the most current edition of The Leadership Challenge (Kouzes \& Posner, 2017) to use throughout the program. Each session was focused on one exemplary leadership behavior. The session designs began with an executive leader joining the cohort for 20 min to share his or her leadership journey and describe how he or she has demonstrated a particular behavior. The action-packed agenda then included various group and mentorship activities designed to allow self-reflection on that session's behavior focus. Each session also focused on the mentorship cycle and helpful strategies to support the mentor-mentee relationship. NMs were not asked to pair up until the end of the second session. This provided the cohort an opportunity to learn more about mentorship and receive the mentorship toolkit with information on how to be a mentor as well as how to meet goals as a mentee. This delay in pairing also allowed the cohort members to get more acquainted with each other. Pairing was done independently with the managers themselves choosing who they felt would best support their mentorship goals. In between the monthly sessions, NMs were asked to read the next chapter in The Leadership Challenge and meet with their mentor at least once prior to the next session. 


\section{Measures and Data Analysis}

The project used the NMPE scale (Warshawsky et al., 2016) to measure intent to stay and job satisfaction before and after the FNMMP. Data were analyzed using SPSS Version 24 to determine if there was any statistical impact on a NM's intent to stay or job satisfaction. Furthermore, this project also used the LPI for the NMs to self-assess their transformational leadership behaviors both before and after the FNMMP. The LPI is a validated tool measuring transformational leadership behaviors (Kouzes \& Posner, 2017). Higher amounts of transformational leadership behaviors have been associated with greater retention of NMs (Cziraki et al., 2014).

Participants in the pilot FNMMP completed the NMPE scale during the first kick-off session using an online survey method, SurveyMonkey, for quick and efficient data collection. Warshawsky et al. (2016) have demonstrated a correlation between this validated tool and NM job satisfaction and intent to stay. Job satisfaction was predicted by three NMPE subscales: Culture of Patient Safety, Constructive Nurse Manager and Director Relationships, and Culture of Generativity. Intent to leave was predicted by the following three subscales: Culture of Generativity, Constructive Nurse Manager and Director Relationships, and Fair and Manageable Workload (p. 506). The tool is a series of questions regarding the practice environment of NMs and is assessed on a Likert-type scale.

Along with the NMPE, all participants in the FNMMP were asked to self-assess their transformational leadership behaviors prior to beginning the program using the LPI developed by Kouzes and Posner (2017). The LPI is an instrument with 30 statements that measure the five practices of exemplary leaders. Internal reliability, as measured by Cronbach's alpha, is strong, with all scales >.75 (Statistic Solutions, 2017). This approach has been used with mentoring activities for NMs as a way to measure progress with leadership competency development (Pedaline et al., 2012). In addition, the LPI results helped guide mentorship needs of the participants and served to measure progress made on transformational leadership behaviors by participants throughout the program.

\section{Results}

For this pilot, 15 out of 35 eligible NMs volunteered to participate in the FNMMP and subsequently completed the program. Seven (46.7\%) of the participants had 12-16 years of nursing experience, and nine participants $60 \%$ listed a bachelor of science in nursing as their highest earned degree. Eight participants (53.3\%) had $\leq 2$ years of managerial experience, 10 (66.7\%10) had been in their current position for $\leq 2$ years, and $13(86.7 \%)$ had no experience being a manager outside of the hospital system.

The FNMMP was composed of six total sessions spanning over 6 months. As vacations and operational needs arose, some participants were unable to attend all six sessions. Overall, 10 participants (67\%) were able to complete at least five out of six of the sessions. Course evaluations were collected at the end of every session and were used to inform the following session design. All ratings in the session evaluations were $\geq 4.4$ on a Likert-type scale ranging from 1 (strong dissatisfaction with the mentoring program) to 5 (strong satisfaction with the mentoring program). The information and materials covered in each session were reported by the participants to be applicable to their position $100 \%$ of the time. The content covered was also felt to be best deployed in the classroom setting as opposed to an online setting $99 \%$ of the time. When evaluating the length of the 2-hr sessions, $98 \%$ of the participants reported that the $2 \mathrm{hrs}$ was "just right" in terms of session length. Table 2 depicts the results of the program evaluations. 
Roth \& Whitehead, 2019

Table 2. Summary of the Formal Nurse Manager Mentorship Program

Session Evaluations

\begin{tabular}{lcc}
\hline Evaluation Questions & Rating $^{\mathbf{a}}$ & \% \\
\hline Session objectives were met & 4.65 & \\
Course materials provided were beneficial & 4.43 & \\
Instructor was knowledgeable about subject matter & 4.6 & \\
Overall satisfaction with the course & 4.52 & \\
Will the information obtained be useful in your & & \\
position? & 100 \\
Yes & 0 \\
No & \\
Do you think this class should be done online or in & \\
person? & \\
Online & & \\
In person & 98.63 \\
This class ran the appropriate length of time & 98 \\
Too long & 2 \\
Just right & \\
Too short & \\
\hline
\end{tabular}

${ }^{a}$ Based on a Likert-type scale ranging from 1 (strong dissatisfaction with the mentoring program)

to 5 (strong satisfaction with the mentoring program).

Once the NMs self-selected their peer mentors, the dyads were asked to meet at least once between sessions to continue to work on the goals they had identified. Meetings could take place online or in person. Eight (53\%) of the participants were able to meet with their peer mentor three or four times during the 6-month program, with four participants (27\%) reporting that they met more than six times.

Overall, 14 (93.3\%) of the participants reported that it was helpful to have a peer mentor to support their career development. The majority $(n=14,93.3 \%)$ also felt that the timing of the sessions was considerate of their work day. The monthly sessions took place from 1 p.m. to 3 p.m. based upon manager feedback, to allow for them to attend to operational demands in the morning hours before allowing time for professional development. All participants $(\mathrm{N}=15,100 \%)$ recommended that the FNMMP continue for future NMs. Open feedback in the last session also revealed that three NMs (20\%) had been on the verge of changing jobs prior to starting the program but felt more confident and supported in their roles afterward and chose to stay. Table 3 describes the overall program mentee evaluations.

Table 3. Summary of Overall Program Evaluation ( $\mathrm{N}=15)$

\begin{tabular}{lcc}
\hline Overall Program Evaluation Questions & $\mathbf{\%}$ & $\boldsymbol{n}$ \\
\hline $\begin{array}{l}\text { Did you find having a peer mentor helpful to your career } \\
\text { development and support needs as a manager? }\end{array}$ & & \\
Yes & 93.3 & 14 \\
No & 6.67 & 1 \\
Was the timing of the class considerate of your workday? & 93.3 & 14 \\
Yes & 6.67 & 1 \\
No & & \\
Would you recommend continuing the program in the & 100 & 15 \\
future? & 0 & 0 \\
Yes & & \\
No &
\end{tabular}


The LPI was developed by Kouzes and Posner (2017) as a way to measure how often an individual is demonstrating the behaviors associated with the exemplary leadership and transformational leadership. The FNMMP used the LPI to help guide mentorship activities and course content including identification of leadership competency opportunity and various NMs' strengths for mentor selection. Measuring the LPI both pre- and postprogram allowed the researcher to determine whether the mentorship activities improved leadership competencies and behaviors through the program. A paired-samples $t$ test $(p=.05)$ was conducted to compare the NMs' pre- and postprogram LPI scores. There were statistically significant differences in the preprogram LPI scores $(M=212.27, S D=37.8)$ and postprogram scores $(M=232.47, S D=25.28)$, $t(14)=-2.83, p=.013$. This demonstrated an increase in leadership competency and transformational leadership behaviors over the course of the FNMMP, which can be clinically significant because it impacts how managers show up to lead their teams. Table 4 depicts the results from the preprogram and postprogram LPI.

Table 4. Leadership Practices Inventory (LPI) Statistical Analysis ( $\mathrm{N}=15$ )

\begin{tabular}{|c|c|c|c|c|c|}
\hline \multirow[b]{2}{*}{ Score } & \multicolumn{2}{|c|}{ Preprogram } & \multicolumn{2}{|c|}{ Postprogram } & \multirow[b]{2}{*}{$\boldsymbol{p}$} \\
\hline & $M$ & $S D$ & M & $S D$ & \\
\hline LPI self-report & 212.27 & 37.80 & 232.47 & 25.58 & .013 \\
\hline
\end{tabular}

Warshawsky et al. (2016) used three NMPE subscales to predict job satisfaction: Culture of Patient Safety, Culture of Generativity, and Constructive Nurse Manager and Director Relationships. The FNMMP used these same subscales to assess whether NMs had greater job satisfaction postprogram (higher scores indicate higher job satisfaction). Using a paired-samples $t$ test, pre- and postprogram means for these three subscales were compared. There was no significant difference between the mean score for the Culture of Patient Safety subscale preprogram $(M=68.47, S D=8.15)$ and postprogram $(M=69.53, S D=10.01), t(14)=-.34, p=.74$. The mean scores for the Constructive Nurse Manager and Director Relationships subscale did not show a significant difference preprogram $(M=25, S D=4.14)$ and postprogram $(M=25.67, S D=6.58), t(14)=-.677$, $p=.67$. However, the Culture of Generativity subscale did show significant differences in scores preprogram $(M=23.20, S D=4.65)$ compared to postprogram $(M=26.20, S D=4.74), t(14)=-2.40, p=.032$. Two of the three subscales did not show statistically significant changes however one subscale did, Culture of Generativity, which can then be interpreted that NM job satisfaction did slightly increase after participating in the FNMMP. It should also be noted that the subscales which did not statistically significantly increase, also did not decrease over the course of six months. Table 5 describes the pre and post program results of the NMPE subscale.

Table 5. Job Satisfaction Statistical Analysis (N = 15)

\begin{tabular}{llllrl}
\hline & \multicolumn{2}{c}{ Preprogram } & \multicolumn{2}{c}{ Postprogram } & \\
\cline { 2 - 5 } NMPE Subscale & \multicolumn{1}{c}{} & $\boldsymbol{S D}$ & $\boldsymbol{M}$ & $\boldsymbol{S D}$ & $\boldsymbol{p}$ \\
\hline Culture of Patient Safety & 68.47 & 8.15 & 69.53 & 10.01 & .74 \\
Constructive Nurse & 25 & 4.14 & 25.67 & 6.58 & .67 \\
$\quad \begin{array}{l}\text { Manager and Director } \\
\text { Relationships }\end{array}$ & & & & & \\
Culture of Generativity & 23.20 & 4.65 & 26.20 & 4.74 & .032 \\
\hline
\end{tabular}

Note. NMPE = Nurse Manager Practice Environment scale. 
Warshawsky et al. (2016) also used three NMPE subscales to predict NMs' intent to leave: Culture of Generativity, Constructive Nurse Manager and Director Relationships, and Fair and Manageable Workload. The FNMMP used these same subscales to assess whether NMs had less intent to leave postprogram (higher scores indicate lower intent to leave).

A paired-samples $t$ test was conducted for the Constructive Nurse Manager and Director Relationships subscale to compare the preprogram scores $(M=25, S D=4.14)$ and the postprogram scores $(M=25.67, S D=$ 6.58 ) and did not result in a significant difference, $t(14)=-.44, p=.67$. The FNMMP did not specifically address how to build constructive relationships with a manager's director, so this result is not surprising.

The mean score for the Fair and Manageable Workload subscale did not show a significant change preprogram $(M=12.60, S D=2.77)$ compared to postprogram $(M=12.93, S D=4.51), t(14)=-.33, p=.75$. Again, as the FNMMP did not specifically address manageable workloads. The Culture of Generativity subscale did show significant differences in scores preprogram $(M=23.20, S D=4.65)$ compared to postprogram $(M=26.20, S D=4.74), t(14)=-2.40, p=.032$. Although two of the three subscales did not experience statistically significant changes, one subscale did, Culture of Generativity. This can indicate that NM intent to leave did slightly decrease after participating in the FNMMP as a result of managers focusing on how to best create culture within their teams. Table 6 describes pre and post program results of the NMPE subscales.

Table 6. Intent to Leave Statistical Analysis ( $\mathrm{N}=15)$

\begin{tabular}{lccccc}
\hline & \multicolumn{2}{c}{ Preprogram } & \multicolumn{2}{c}{ Postprogram } & \\
\cline { 2 - 5 } NMPE Subscale & $\boldsymbol{M}$ & $\boldsymbol{S D}$ & $\boldsymbol{M}$ & $\boldsymbol{S D}$ & $\boldsymbol{p}$ \\
\hline $\begin{array}{l}\text { Constructive Nurse } \\
\quad \begin{array}{l}\text { Manager and Director } \\
\text { Relationships }\end{array}\end{array}$ & 25 & 4.14 & 25.67 & 6.58 & .67 \\
$\begin{array}{l}\text { Fair and Manageable } \\
\quad \text { Workload }\end{array}$ & 12.6 & 2.77 & 12.93 & 4.51 & .75 \\
$\quad$ Culture of Generativity & 23.20 & 4.65 & 26.20 & 4.74 & .032 \\
\hline
\end{tabular}

Note. NMPE = Nurse Manager Practice Environment scale.

Turnover rates were another outcome metric that were monitored prior to and after the FNMMP. The hospitals' preprogram (i.e., 2017) NM turnover rates were $25.8 \%$, whereas postprogram (i.e., 2018) turnover rates were $11.9 \%$. This decrease was apparent despite both hospitals going through union contract bargaining, which inherently caused tension between unit managers and staff and a chief nurse leadership vacancy-both of which caused tremendous stress at the NM level.

\section{Discussion}

The purpose of this project was to evaluate whether a formal mentorship program for NMs impacted their job satisfaction and intent to stay. Without having a dedicated process to help NMs engage in a mentorship relationship, many managers go without that support despite the evidence supporting mentorship (Cziraki et al., 2014). This study contributes to the evidence supporting the need for sustainable NM mentorship opportunities as well as the quantifiable benefits of such mentorship. Participation in the FNMMP resulted in NMs increasing their own leadership competencies over time with the guidance of a peer mentor. NMs in this program demonstrated an increase in job satisfaction and decrease in intent to leave. At the organizational level, the FNMMP resulted in a reduction of NM turnover compared to the previous year, which allowed for 
continued unit-level performance and minimal unit disruption with leadership changes. The FNMMP also contributed to a supportive environment and collegiality between the two hospitals and among the NMs. These findings were consistent with those of other studies on NM mentorship, such as the Organization of Nurse Executives of New Jersey statewide mentorship program (Vitale, 2018).

\section{Lessons Learned}

Mentoring in nursing has long been identified as a way to retain nurses and recently there is a growing body of evidence that mentorship for NMs is equally important (Vitale, 2018). One lesson learned was the importance of purposeful conversations and activities that promoted a goal-oriented mentor-mentee relationship. The project used an evidence-based approach to facilitate mentorship relationships using the exemplary leadership framework described by Kouzes and Posner (2017). Pedaline et al. (2012) described using this framework to guide mentorship activities in a group setting. In addition, Gooch (2017) described using it to guide mentorship with new and seasoned chief nurse executives. The evidence supported the use of this framework. The participating NMs found applying the components of transformational leadership useful in their mentorship activities. Without purposeful connection, mentorship relationships may dissolve.

\section{Limitations}

One of the limitations for this project was the small sample size. Although one third of the NMs across both hospitals participated in the program, the sample size was too small to determine if results would be consistent in a larger population. Our recommendation is to conduct this study using upcoming mentorship programs to see if results are the same. Another limitation is that the organization was undergoing national contract bargaining during the majority of this program. Labor negotiations for these hospitals created a tense work environment that the NMs were not accustomed to. Combined with the labor negotiations was the sudden departure of one site's chief nurse executive, which also had an impact on the nurse leaders. These events could have affected the job satisfaction and intent to leave of the participants. Noteworthy, however, is that despite these events, job satisfaction, intent to stay, and LPI scores did demonstrate improvement after mentorship program completion.

\section{Future Applications}

The FNMMP demonstrated a sustainable approach to providing mentorship for NMs with measurable outcomes. However, for future applications, recommendations for improvement include assessing the quality of mentorship relationships with more qualitative inquiry such as interviews and focus groups.Understanding how well the dyads are communicating and connecting can inform future programs.

\section{References}

Cziraki, K., McKey, C., Peachey, G., Baxter, P., \& Flaherty, B. (2014). Factors that facilitate registered nurses in their first-line nurse manager role. Journal of Nursing Management, 22, 1005-1014. https://doi.org/10.1111/jonm.12093

Cadmus, E., \& Johansen, M. L. (2012, October). The time is now: Developing a nurse manager residency program. Nursing Management, 43, 19-24. http://dx.doi.org/10.1097/01.NUMA.0000419448.52255.6c 
DeCampli, P., Kirby, K. K., \& Baldwin, C. (2010, April-June). Beyond the classroom to coaching: Preparing new nurse managers. Critical Care Nursing Quarterly, 33, 132-137. http://dx.doi.org/10.1097/CNQ.obo13e3181d913db

Djukic, M., Jun, J., Kovner, C., Brewer, C., \& Fletcher, J. (2017, April-June). Determinants of job satisfaction for novice nurse managers employed in hospitals. Healthcare Management Review, 42, 172-183. https://doi.org/10.1097/HMR.0000000000000102

Fischer, S. A. (2016). Transformational leadership in nursing: A concept analysis. Journal of Advanced Nursing, 72, 2644-2653. https://doi.org/10.111/jan.13049

Gooch, P. (2017, October ). Creating a mentoring culture for new and seasoned chief nurse executives in a health system. Nurse Leader, 15, 341-344.

Hewko, S. J., Brown, P., Fraser, K. D., Wong, C. A., \& Cummings, G. G. (2015, November). Factors influencing nurse managers' intent to stay or leave: A quantitative analysis. Journal of Nursing Management, 23, 1058-1066. https://doi.org/10.1111/jonm.12252

Kalbfleisch, P. J. (2002, February). Communicating in mentoring relationships: A theory for enactment. Communication Theory, 12, 63-69. https://doi.org/10.1111/j.1468-2885.2002.tbo0259

Kalbfleisch, P. J. (2008). Mentoring enactment theory: Describing, explaining, and predicting communication in mentoring relationships. In B. R. Ragins \& K. E. Kram (Eds.), The handbook of mentoring at work: Theory, research, and practice (pp. 499-518). Thousand Oaks, CA: Sage. https://doi.org/10.4135/9781412976619.n20

Kelly, L., Wicker, T. L., \& Gerkin, R. D. (2014, March). The relationship of training and education to leadership practices in frontline nurse leaders. The Journal of Nursing Administration, 44, 158-163. https://doi.org/10.1097/NNA.0000000000000044

Keys, Y. (2014). Looking ahead to our next generation of nurse leaders: Generation X nurse managers. Journal of Nursing Management, 22, 97-105. https://doi.org/10.1111/jonm.12198

Kouzes, J. M., \& Posner, B. Z. (2017). The leadership challenge (6th ed.). Hoboken, NJ: John Wiley.

Mansson, D. H., \& Myers, S. A. (2012, October). Using mentoring enactment theory to explore the doctoral study-adviser mentoring relationship. Communication Education, 61, 309-334. https://doi.org/10.1080/03634523.2012.708424

Miltner, R. S., Jukkala, A., Dawson, M. A., \& Patrician, P. A. (2015). Professional development needs of nurse managers. Journal of Continuing Education in Nursing, 46, 252-258. http://dx.doi.org/10.3928/00220124-20150518-01

Oregon Center for Nursing. (2016). The demand for nursing professionals in Oregon in 2016. Washington, DC: Government Printing Office. Retrieved from http://oregoncenterfornursing.org/wpcontent/uploads/2014/09/2016-OCN-TheDemandforNursingProfessionals-Web-NEW.pdf

Pedaline, S. H., Wolf, G., Dudjak, L., Lorenz, H., McLaughlin, M., \& Ren, D. (2012, September). Preparing exceptional leaders. Nursing Management, 43, 38-44. https://doi.org/10.1097/01.NUMA.0000416401.45485.f6

Rich, M., Kempin, B., Loughlin, M. J., Vitale, T. R., Wurmser, T., \& Thrall, T. H. (2015, February). Developing leadership talent: A statewide nurse leader mentorship program. The Journal of Nursing Administration, 45, 63-66.

Sherman, R. O., Patterson, P., Avitable, T., \& Dahle, J. (2014, July-August). Perioperative nurse leader perspective on succession planning: A call to action. Nursing Economic $\$$, 34, 186-203. 
Statistic Solutions. (2017). Leadership Practices Inventory (LPI). Retrieved from http://www.statisticssolutions.com/leadership-practices-inventory-lpi/

Vitale, T. R. (2018, February). Nurse leader mentorship. Nursing Management, 49, 8-10. https://doi.org/10.1097/01.NUMA.0000529932.89246.ab

Warshawsky, N., Rayens, M., Stefaniak, K., \& Rahman, R. (2013, July). The effect of nurse manager turnover on patient fall and pressure ulcer rates. Journal of Nursing Management, 21, 725-732. https://doi.org/10.1111/jonm.12101

Warshawsky, N., Wiggins, A., \& Rayens, M. (2016, October). The influence of the practice environment on nurse managers' job satisfaction and intent to leave. The Journal of Nursing Administration, 46, 501-507. https://doi.org/10.1097/NNA.0000000000000393

Weaver-Moore, L., Sublett, C., \& Leahy, C. (2016). Nurse managers' insights regarding their role highlight the need for practice changes. Applied Nursing Research, 30, 98-103. https://doi.org/10.1016/j.apnr.2015.11.006

Westcott, L. (2016). How coaching can play a key role in the development of nurse managers. Journal of Clinical Nursing, 25, 2669-2677. http://dx.doi.org/10.1111/jocn.13315

Wong, C. (2015). Connecting nursing leadership and patient outcomes: State of the science. Journal of Nursing Management, 23, 275-278. https://doi.org/10.1111/jonm.12307 and international workforce issues, quality improvement projects, evidence-based practice initiatives, nursing research studies, interprofessional practice, educational issues, telehealth, improvements in technology, and the impact of social change in society. 